\title{
Single-molecule level rare events revealed by dynamic surface-enhanced Raman spectroscopy
}

Cheng Zong ${ }^{1 \dagger}$, Chan-juan Chen ${ }^{1}$, Xiang Wang ${ }^{1}$, Pei Hu ${ }^{1}$, Guo-kun Liu² ${ }^{*}$, and Bin $\operatorname{Ren}^{1 *}$

1. State Key Laboratory of Physical Chemistry of Solid Surfaces, Collaborative Innovation Center of Chemistry for Energy Materials (iChEM), College of Chemistry and Chemical Engineering, Xiamen University, Xiamen, China

2. State Key Laboratory of Marine Environmental Science, Fujian Provincial Key Laboratory for Coastal Ecology and Environmental Studies, Center for Marine Environmental Chemistry \& Toxicology, College of the Environment and Ecology, Xiamen University, Xiamen 361102, China

*Corresponding author: guokunliu@xmu.edu.cn, bren@xmu.edu.cn

Figure S1. Scheme of data analysis

Figure S2. The autocorrelation curve from SERS intensity trajectory.

Figure S3. The potential dependent SERS spectra of R6G

Figure S4. The autocorrelation curves the corresponding popular and rare events

Figure S5. The histogram of the number of rare event

Table S1. The reading time and noise of EMCCD under different working mode

Table S2. SNR, signal and noise of spectra obtained from the $10^{-7} \mathrm{M}$ R6G sample

Table S3. SNR, signal and noise of spectra obtained from the $10^{-7} \mathrm{M}$ R6G with $10^{-2} \mathrm{M} \mathrm{Cl}^{-}$sample

Supporting information 1 . The quantitative analysis of the contribution of different noise source 

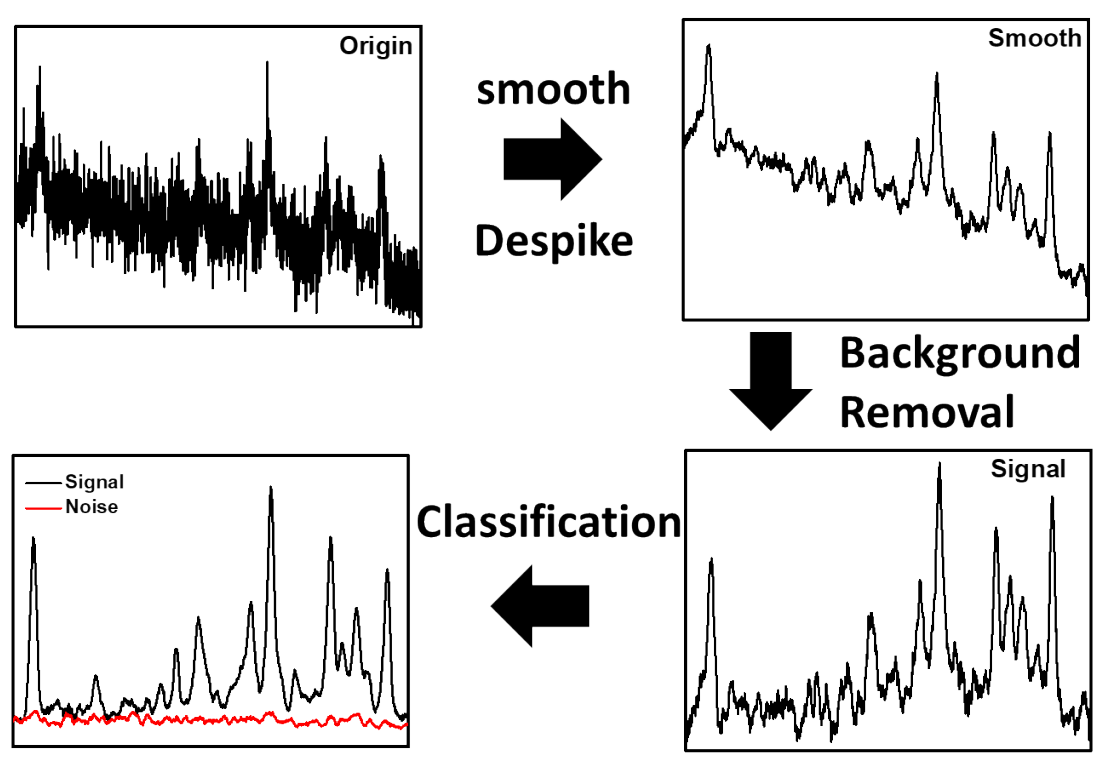

Fig. S1. Scheme of data analysis. Original data were denoised by Savitzky-Golay smooth and despiked the cosmic ray. The back-ground were subtracted by a penalized least-squares approach. 

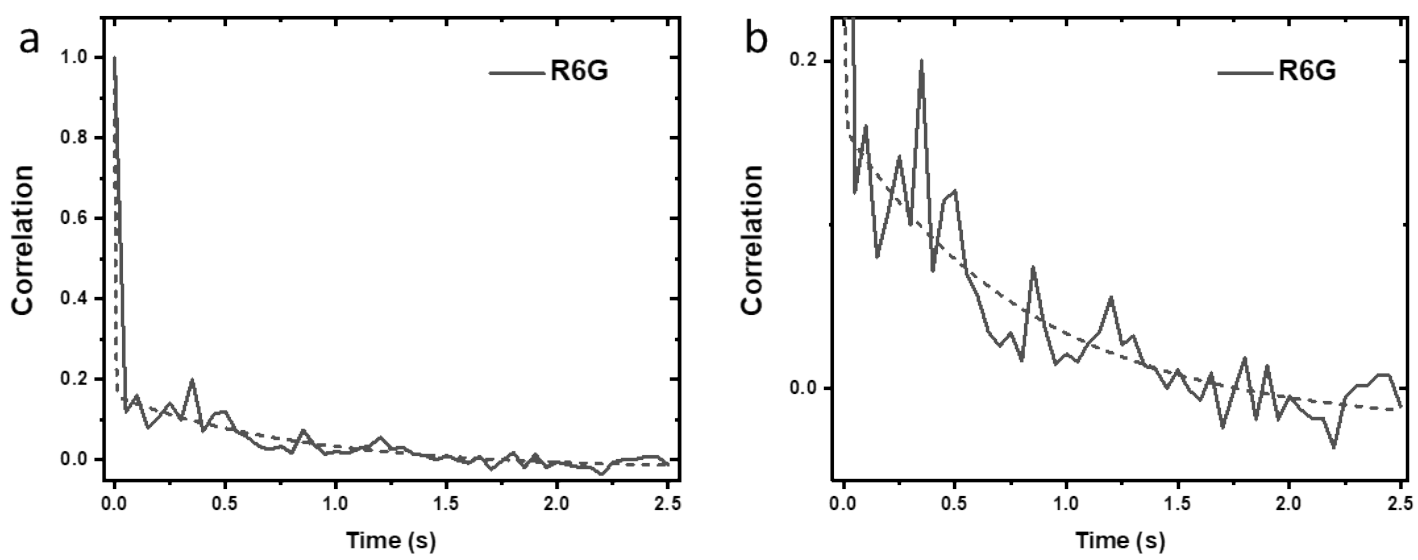

Figure S2. Autocorrelation curve of Raman intensity of $1645 \mathrm{~cm}-1$ in peak extracted from the heatmap in Fig. 1a starting from tau $=0 \mathrm{~s}$ to tau $=2.5 \mathrm{~s} \mathrm{(a)}$ and the zoom-in curve from tau $=0.05 \mathrm{~s}$ to tau $=2.5 \mathrm{~s}$ (b). The autocorrelation curve shows exponential decay behavior, manifesting temporal SERS fluctuations of few R6G molecules. The autocorrelation coefficient at $0.05 \mathrm{~s}$ is $\sim 0.15$ and approaches quickly to zero after $1 \mathrm{~s}$, which indicate little cross-talking between the consecutive SERS spectra under the current condition. 

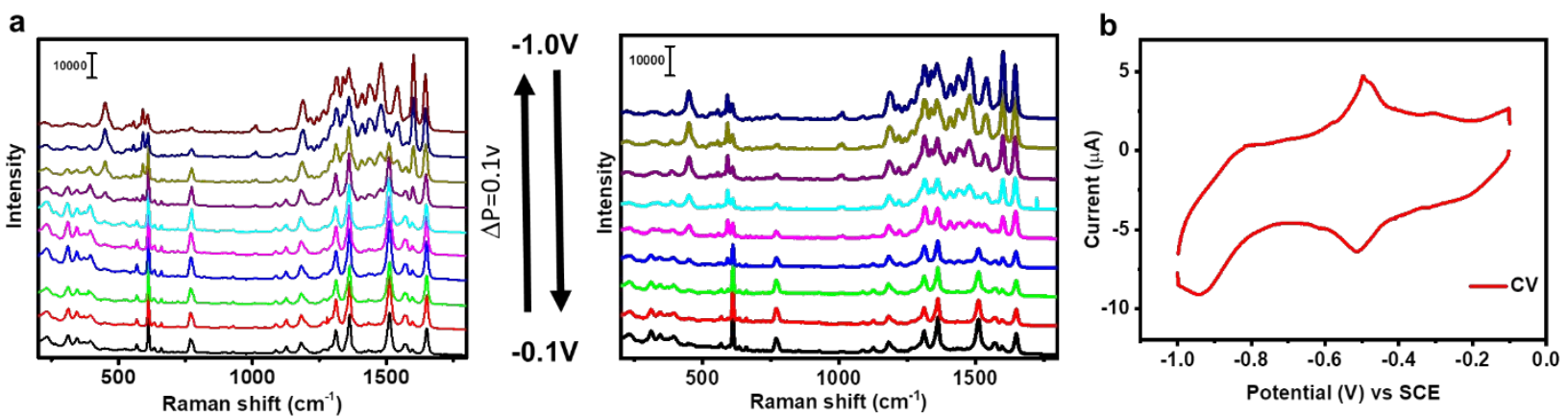

Fig. S3 (a) Potential dependent SERS spectra of R6G adsorbed on a rough Ag electrode. left: forward potential scan from $-0.1 \mathrm{~V}(\mathrm{OCP})$ to $-1.0 \mathrm{~V}$. Right: backward potential scan from $-1.0 \mathrm{~V}$ to $-0.1 \mathrm{~V}$. (b) cyclic voltammetry of R6G on rough Ag electrode. At $-0.7 \mathrm{~V}$, the intensity of ratio of $1575 \mathrm{~cm}^{-1}$ to 1600 $\mathrm{cm}^{-1}$ was reversed. And the cyclic voltammetry result presents that a reversible peak at $-0.66 \mathrm{~V}$ which is the balance potential of $\mathrm{R} 6 \mathrm{G}^{+}$and $\mathrm{R} 6 \mathrm{G}^{0}$ on Ag electrode. The potential-dependent result indicates the redox process of $\mathrm{R} 6 \mathrm{G}$ on $\mathrm{Ag}$ electrode is reversed. 

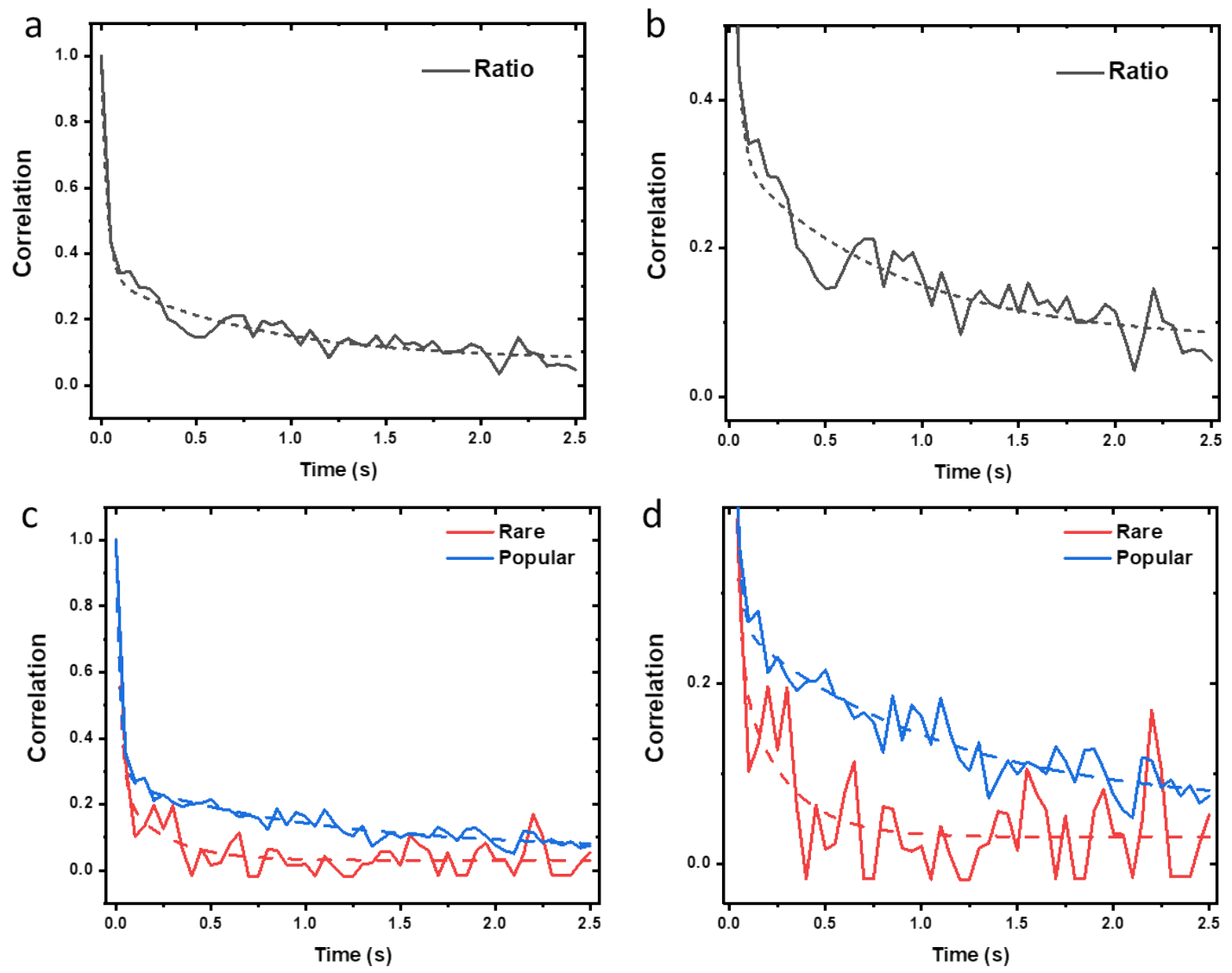

Figure S4. The autocorrelation curve of rare and popular events. a) The autocorrelation curve from Raman intensity ratio of the $1575 \mathrm{~cm}^{-1}$ and $1600 \mathrm{~cm}^{-1}$ peaks extracted from the heatmap in Fig. 2a. b) The zoom-in curve of (a) from tau $=0.05 \mathrm{~s}$ to tau $=2.5 \mathrm{~s} \mathrm{c}$ ) The autocorrelation curves of the corresponding popular and rare events. d) The zoom-in curve of (c) from tau $=0.05 \mathrm{~s}$ to tau=2.5 s c). Note: the autocorrelation curve of rare event is calculated by setting the popular event as zero and vice versa.

As shown in Figure S4a\&b, the autocorrelation coefficient quickly decreased from 0.4 to 0.2 in 0.5 $\mathrm{s}$ and then fluctuated at 0.1 after $1 \mathrm{~s}$. Compared with that in Figure S2, t, in the presence of $\mathrm{Cl}^{-}$, the cross talking behavior between two neighbor SERS spectra is strengthened, but not for the nonadjacent SERS spectra. As shown in Figure S4c\&d, the decay of the rare event is much faster than that of the popular one, which clearly implies negligible correlation between the consecutive SERS spectra of R6G. The rare event shows the typical single molecule behavior. 


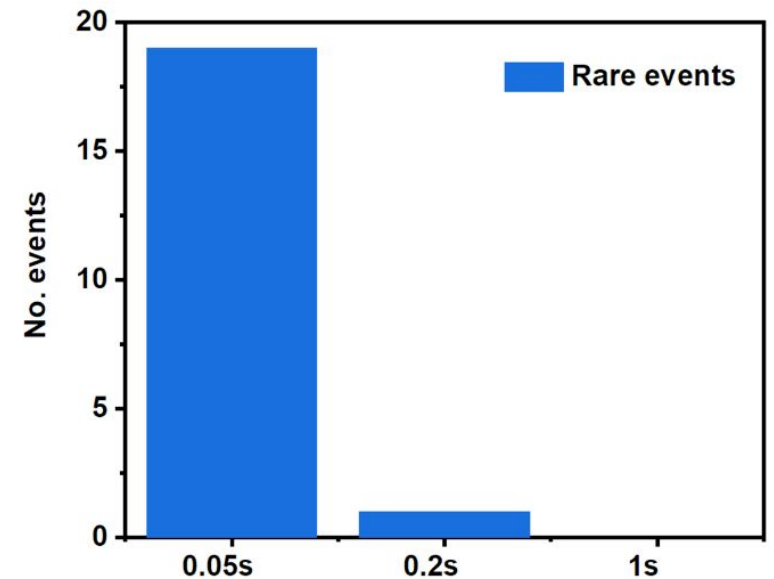

Fig. S5. Histogram of the number of rare event for integration time of $0.05 \mathrm{~s}, 0.2 \mathrm{~s}$, and $1 \mathrm{~s}$, respectively 
Table S1 the reading time and noise of EMCCD

\begin{tabular}{ccccc}
\hline Readout channel & Readout mode & Actual time* & Dead time & Noise $^{\#}$ \\
\hline \multirow{2}{*}{ EM } & FVB & $50.00 \mathrm{~ms}$ & $0.00 \mathrm{~ms}$ & 6.43 \\
\cline { 2 - 5 } & ST & $55.26 \mathrm{~ms}$ & $5.26 \mathrm{~ms}$ & 5.98
\end{tabular}

* All exposure time is set as $50 \mathrm{~ms}$.

\#all data were collected without illumination. 
Table S2 SNR, signal and noise of spectra obtained from the $10^{-7} \mathrm{M}$ R6G sample

\begin{tabular}{cccc}
\hline Exposure time (s) & SNR & Signal & Noise \\
\hline 0.05 & 6.1 & 58.1 & 9.5 \\
0.2 & 4.0 & 14.2 & 3.5 \\
1 & 1.7 & 3.5 & 2.1 \\
5 & 1.0 & 0.8 & 0.7 \\
\hline
\end{tabular}

* The exposure time of background and impurity is $0.05 \mathrm{~s}$. 
Table S3 SNR, signal and noise of spectra obtained from the $10^{-7} \mathrm{M}$ R6G with $10^{-2} \mathrm{M} \mathrm{Cl}^{-}$sample

\begin{tabular}{cccc}
\hline Exposure time (s) & SNR & Signal & Noise \\
\hline 0.05 & 7.4 & 126.2 & 17.0 \\
0.2 & 14.1 & 117.9 & 8.4 \\
1 & 24.6 & 130.0 & 5.3 \\
5 & 33.7 & 105.9 & 3.1 \\
\hline
\end{tabular}




\section{Supporting information 1 . The quantitative analysis of the contribution of different noise source}

Here, we quantitatively analyze the relative contribution of difference noise sources to the SERS spectrum, including unavoidable noise $\left(\sigma_{N}\right)$ from dark noise $\left(\sigma_{d}\right)$ and readout noise $\left(\sigma_{r}\right)$, and unwanted noise $\left(\sigma_{u}\right)$ from background and impurities.

We first compare the relative contribution of the dark noise and readout noise under the current instrument condition in two readout modes, full vertical binning (FVB) and single track (ST). In FVB mode, the signal along all the 200 pixel in each vertical line are combined together in one horizontal CCD register before readout. Therefore, the noise of FVB is the sum of the dark noise from all 200 lines and the readout noise. Whereas, the noise of ST is the sum of the dark noise and the readout noise from a single line since only one line is selected for readout. As shown in Table S1, with the EMCCD mode under the current condition, the noise $\left(\sigma_{\mathrm{N}}\right)$ of FVB mode is 6.43 , which increases by $7 \%$ in comparison with that of ST (5.98). Based on the following equation

$$
\begin{gathered}
\sigma_{N}^{2}=\sigma_{d}^{2}+\sigma_{r}^{2} \text { (Eq. S1) } \\
\sigma_{T}^{2}=\sigma_{u}^{2}+\sigma_{d}^{2}+\sigma_{r}^{2} \text { (Eq. S2) }
\end{gathered}
$$

where, $\sigma_{N}$ is the measured noise without any illumination. $\sigma_{T}$ is the total noise of the SERS spectrum. Therefore, the readout and dark noises can be calculated as 5.977 and 0.17 per line per $50 \mathrm{~ms}$, respectively, which indicates that readout noise is the dominated noised without any illumination.

The background in our experiment may originate from photoluminescence of nanostructured SERS substrate or fluorescence of R6G in solution, which shows a broad feature in spectrum. The background signal is consistent and slightly fluctuates for all spectra, being demonstrated in Figure S6a. The noise from background could be calculated as the standard deviation of each spectrum. Taken these six spectra as an example, the noise from background and the total noise are fluctuated in the range of 2.86 5.25 (average: 4.19) and 7.04 8.30 (average: 7.71), respectively (Table S4).

The sharp features exhibited by the impurity spectrum (Figure S6b) is different from that of R6G. The impurity may originate from by-products in nanoparticles synthesis or other species in the solution. The appearance of impurity signal is random which is determined by the dynamic adsorption of impurity. Therefore, the noise from impurity is calculated as the standard deviation of the spectrum containing random impurity signal. Taken these six spectra as an example, the noise from impurity and the total noise are fluctuated in the range of 5.04 19.59 (average: 11.95) and 8.17 20.62 (average: 13.81), respectively (Table S4).. Obviously, the noise from impurities is the dominated noise contributing to the 
total noise, and the accompanied interference to the trace detection of weak-binding targets could be effectively alleviated by the dynamic SERS strategy.

\section{Background}

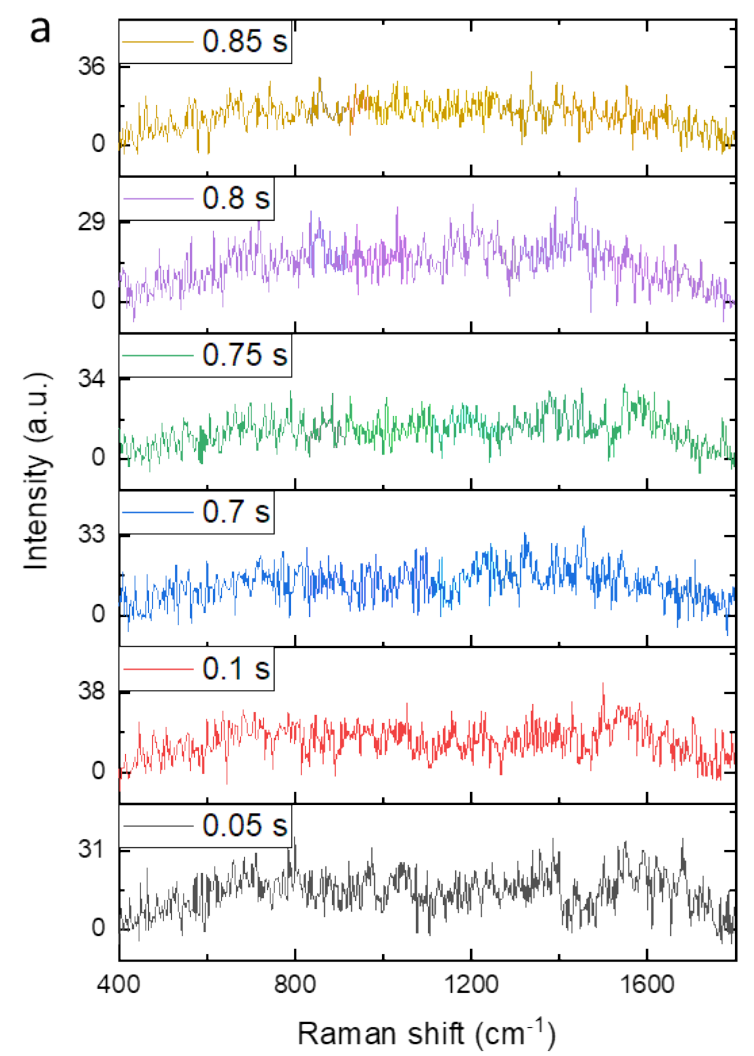

Impurity

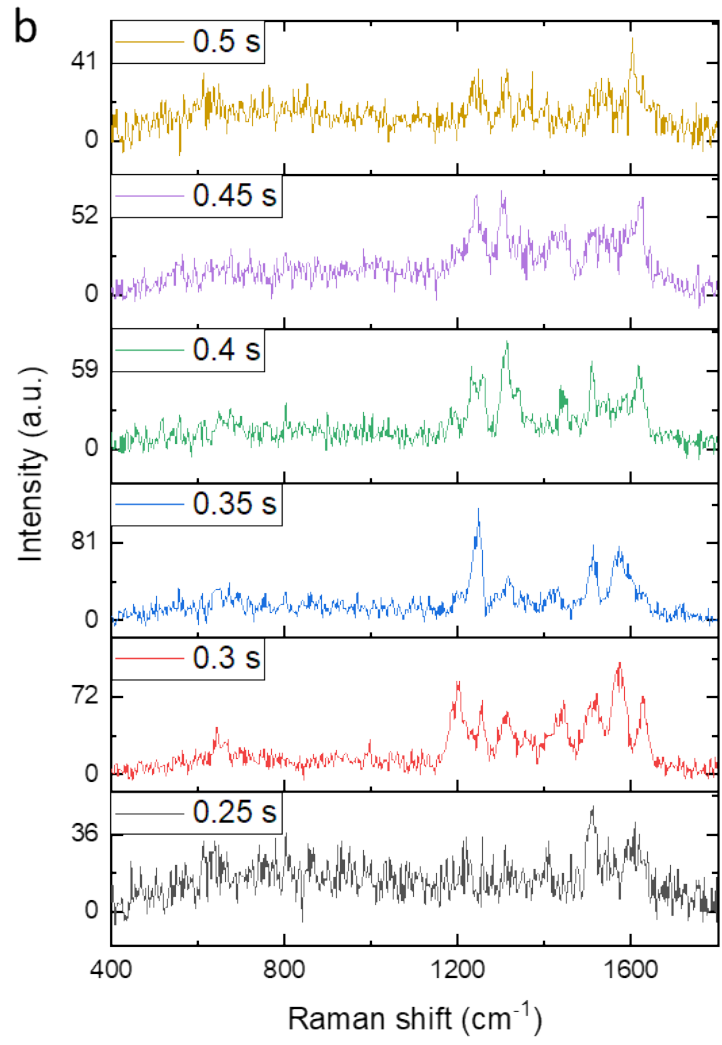

Figure S6 Typical SERS spectra dominated by background (a) and impurities (b) with an acquisition time of $0.05 \mathrm{~s}$. 
Table S4. The noise contribution from background and impurity

\begin{tabular}{ccccc}
\hline $\begin{array}{c}\text { Corresponding } \\
\text { spectra }\end{array}$ & $\begin{array}{c}\text { Total noise of } \\
\text { background spectra }\end{array}$ & $\begin{array}{c}\text { The noise from } \\
\text { background }\end{array}$ & $\begin{array}{c}\text { Total noise of } \\
\text { impurity spectra }\end{array}$ & $\begin{array}{c}\text { The noise } \\
\text { from impurity }\end{array}$ \\
\hline Black & 8.08 & 4.89 & 9.16 & 6.52 \\
Red & 8.30 & 5.25 & 20.62 & 19.59 \\
Blue & 7.58 & 4.03 & 16.39 & 15.08 \\
Green & 7.19 & 3.23 & 14.45 & 12.94 \\
Purple & 8.06 & 4.87 & 14.08 & 12.53 \\
Yellow & 7.04 & 2.86 & 8.17 & 5.04 \\
\hline Average & 7.71 & 4.19 & 13.81 & 11.95 \\
\hline
\end{tabular}

